UDC 510.225

\author{
A. N. ANIKIEV
}

\title{
PLANE DOMAINS WITH SPECIAL CONE CONDITION
}

\begin{abstract}
The paper considers the domains with cone condition in $\mathbb{C}$. We say that domain $G$ satisfies the (weak) cone condition, if $p+V(e(p), H) \subset G$ for all $p \in G$, where $V(e(p), H)$ denotes right-angled circular cone with vertex at the origin, a fixed solution $\varepsilon$ and a height $H, 0<H \leq \infty$, and depending on the $p$ vector $e(p)$ axis direction. Domains satisfying cone condition play an important role in various branches of mathematic (e.g. [1], [2], [3] (p. 1076), [4]). In the paper of P. Liczberski and V. V. Starkov, $\alpha$-accessible domains were considered, $\alpha \in[0,1)$, - the domains, accessible at every boundary point by the cone with symmetry axis on $\{p t: t>1\}$. Unlike the paper of P. Liczberski and V.V. Starkov, here we consider domains, accessible outside by the cone, which symmetry axis inclined on fixed angle $\phi$ to the $\{p t: t>1\}, 0<\|\phi\|<$ $<\pi / 2$. In this paper we give criteria for this class of domains when the boundaries of domains are smooth, and also give a sufficient condition when boundary is arbitrary. This article is the full variant of [5], published without proofs.
\end{abstract}

Key words: $(\alpha, \beta)$-accessible domain, cone condition

2010 Mathematical Subject Classification: 26A21

1. Introduction. In [6] (see also [7]) $\alpha$-accessible domain, $\alpha \in[0,1$ ), were introduced and studied. A domain $\Omega \subset \mathbb{R}^{n}, 0 \in \Omega$, is called $\alpha-$ accessible, if for every point $p \in \partial \Omega$ there exists a number $r=r(p)>0$ such that the cone

$$
K_{+}(p, \alpha, r)=\left\{x \in \mathbb{R}^{n}:\left(x-p, \frac{p}{\|p\|}\right) \geq\|x-p\| \cos \left(\frac{\alpha \pi}{2}\right),\|x-p\| \leq r\right\}
$$

is included in $\Omega^{\prime}=\mathbb{R}^{n} \backslash \Omega$. 
In particular, in [6] the authors proved that $\alpha$-accessible domains are bounded and satisfy cone condition when $\alpha \in(0,1)$ and $e(p)=-p$. This condition of radiality axis of symmetry applies significant limitation on $\Omega$.

The paper consider the case, when the axis of cone symmetry is lies on ray, containing 0 and $p$, and crosses the cone.

Definition 1. A domain $\Omega \subset \mathbb{C}, 0 \in \Omega$, is called $(\alpha, \beta)$-accessible, $\alpha, \beta \in$ $\in[0,1)$, if for every point $p \in \partial \Omega$ there exists a number $r=r(p)>0$ so that the cone

$$
K_{+}(p, \alpha, \beta, r)=\left\{z \in \mathbb{C}:-\frac{\beta \pi}{2} \leq \arg (z-p)-\arg (p) \leq \frac{\alpha \pi}{2},|z-p| \leq r\right\}
$$

is included in $\Omega^{\prime}=\mathbb{C} \backslash \Omega$.

Let us denote $\alpha_{0}=\min (\alpha, \beta), \beta_{0}=\max (\alpha, \beta)$. Note that the class of $(\alpha, \beta)$-accessible domains is intermediate between $\alpha_{0}-$ and $\beta_{0}$-accessible classes.

The purpose of this paper is to discuss the failure of condition $e(p)=$ $=-p$, when the angle (let us denote it by $\phi$ ) of inclination axis of symmetry to the ray $\{p t: t>0\}$ is a constant.

It is interesting to figure out how the properties of domains with this inclination will be changed. This problem is very difficult for large values of $\phi\left(\phi>\frac{\pi}{2}\right)$ even in the case of permanent angle $\phi$. In this case, the methods by which the results were obtained in [6] are no longer applicable.

This work does not provide a complete description of these areas - this task is too complex, but at this stage it's unable to get rid of condition $e(p)=-p$ and replace it by the condition of the Def. 1 , when $\phi$ is constant.

Let's introduce some other definitions.

Definition 2. We call a domain $\Omega$ starlike with respect to 0 if for every point $z \in \Omega$ segment $[0, z]$ is contained in $\Omega$.

Definition 3. We call a domain $\Omega$ a strong-starlike with respect to 0 if $[0, p] \cap \partial \Omega=p$ for every point $p \in \partial \Omega$.

\section{Case of arbitrary boundary.}

Theorem 1. If the domain $\Omega$ is $(\alpha, \beta)$-accessible, $\alpha, \beta \in(0,1)$, then for each point $p \in \partial \Omega$ and for every $\varepsilon \in(0, \min (\alpha, \beta))$ there exists a number $\rho$ such that $\rho(p)>0$ and the cone $K_{-}(p, \alpha-\varepsilon, \beta-\varepsilon, \rho) \subset \Omega$, where

$$
K_{-}(p, \alpha-\varepsilon, \beta-\varepsilon, \rho)=
$$


$=\left\{z \in \mathbb{C}:-\frac{(\beta-\varepsilon) \pi}{2}<\arg (z-p)-\arg (-p)<\frac{(\alpha-\varepsilon) \pi}{2},|z-p|<\rho\right\}$.

Proof. Suppose not. Then there exists a point $p \in \partial \Omega$ such that

$$
K_{-}(p, \alpha-\varepsilon, \beta-\varepsilon, \rho) \cap \Omega^{\prime} \neq \varnothing
$$

for $\rho>0$ and $\varepsilon \in(0, \min (\alpha, \beta))$. This shows that there exists a sequence of points such that $\left\{w_{m}\right\} \in K_{-}(p, \alpha-\varepsilon, \beta-\varepsilon, \rho) \cap \Omega^{\prime}$, and $z_{m} \rightarrow p$ as $m \rightarrow \infty$. Consider $C\left(p,\left|w_{m}\right|\right)$ - circle with center of $p$ and radius $\left|w_{m}\right|$. This circle intersects the segment $[0, p)$. Associate point $w_{m}$ with those, which are obtained as a result of intersection $C\left(p,\left|z_{m}\right|\right) \cap[0, p)$ with arc of circle, are placed in $\operatorname{Int}\left(K_{-}(p, \alpha-\varepsilon, \beta-\varepsilon, \rho)\right)$. As $\partial \Omega$ is connected, this arc of circle intersects the bound of $\Omega$. Thus we get sequence of points lying on bound of $\Omega$, which converges to $p$. Let us denote this sequence $\left\{w_{m}\right\}$.

Denote by $l(\theta)$ the ray, starting from 0 and passing through the segment $[0, \mathrm{p}]$ with angle $\theta$. In [6, proof of Theorem 1] was proved existence of $l_{\theta} \cap \partial \Omega$ and a unique. Thus, $\Omega$ is a strong-starlike domain.

Introduce a function $r=r(\theta)$, the distance from 0 to the point of intersection of the ray $l(\theta)$ with $\partial \Omega$. From [6, proof of Theorem 1] it follows that $r(\theta)$ is continuous.

There exists $n \in \mathbb{N}$ such that for all $m>n$

$$
\left|\arg \left(w_{m}\right)-\arg (p)\right|<\frac{\varepsilon \pi}{2} .
$$

Denote by $\phi_{m}=\arg \left(w_{m}\right)-\arg (p), \phi_{m} \in(-\pi ; \pi]$.

Now let us consider that $L$ is part of $\partial \Omega$, lying between $l(0)$ and $l\left(\phi_{m}\right)$.

As $w_{m} \in \partial \Omega$, then for it exists cone $K_{+}\left(w_{m}, \alpha, \beta, r_{m}\right) \subset \Omega, r_{m}>0$.

Consider two ways:

1) Let $\phi_{m}>0$. Draw a line through $w_{m}$ parallel those sides of cone $K_{-}(p, \alpha-\varepsilon, \beta-\varepsilon, \rho)$, which intersect $l\left(\phi_{m}\right)$. This line intersects segment $[0, p]$ at the point $A:|A|<|p|$. The same side of cone $K_{+}\left(w_{m}, \alpha, \beta\right)$ intersects the segment $[0, p]$ at the point $B:|B|<|A|$. This is true, when:

$$
\frac{\beta \pi}{2}>\frac{(\beta-\varepsilon) \pi}{2}+\left|\phi_{m}\right|
$$

2) Let now $\phi_{m} \leq 0$. By similar reasoning, we obtain:

$$
\frac{\alpha \pi}{2}>\frac{(\alpha-\varepsilon) \pi}{2}+\left|\phi_{m}\right| .
$$


From $(*)$ it follows that for sufficiently large number $m$ the inequalities (1) and (2) hold. By the fact, that $\Omega$ is $(\alpha, \beta)$-accessible and

$$
K_{+}\left(w_{m}, \alpha, \beta, r_{m}\right) \cap \Omega=\varnothing
$$

we have $L \cap K_{+}\left(w_{m}, \alpha, \beta, r_{m}\right)=\varnothing$.

Consider $L \cap\left[w_{m}, B\right]$. Let $w_{0}$ is closest to $B$ point of intersection $L \cap\left[w_{m}, B\right]$. Denote by $\theta_{0}=\arg \left(w_{0}\right)-\arg (p)$, the angle between the ray $l\left(\theta_{0}\right)$, going from 0 through point $w_{0}$, and the segment $[0, \mathrm{p}]$.

As $w_{0} \in \partial \Omega$, for it exists cone $K_{+}\left(w_{0}, \alpha, \beta, \delta\right)$ such that

$$
K_{+}\left(w_{0}, \alpha, \beta, \delta\right) \cap \Omega=\varnothing
$$

for sufficiently small $\delta>0$. The side of cone $K_{+}\left(w_{0}, \alpha, \beta\right)$ intersects the segment $[0, p]$ in point $C$ in the way that $|C|<|B|$. It follows from the fact that cone $K_{+}\left(w_{0}, \alpha, \beta\right)$ obtains from cone $K_{+}\left(w_{m}, \alpha, \beta\right)$ by turning an angle $\left(\theta_{0}-\phi_{m}\right)$.

For $L$ to connect $w_{0}$ and $p$, it must either intersect $\left(w_{0}, B\right)$, or intersect segment $[0, p]$. None of both is possible. Indeed, by the definition of $w_{0}$, $L$ can't intersect the segment $\left(w_{0}, B\right)$. On the other hand, by virtue of an unambiguous definition $r(\theta), \partial \Omega$ can't contain the radial segments $[6$, Theorem 1], so it doesn't contain the points from $[0, p)$. Hence we get a contradiction with the fact, that theorem is wrong. The proof is complete now.

Theorem 2. If $\Omega$ is $(\alpha, \beta)$-accessible, then for every point $p \in \partial \Omega$ and for every fixed $\alpha, \beta \in[0,1)$ unbounded cone $K_{+}(p, \alpha, \beta, \infty):=K_{+}(p, \alpha, \beta)$ belongs to $\mathbb{C} \backslash \Omega=\Omega^{\prime}$.

Proof. Suppose that the theorem is wrong. Then there is a point $p \in \partial \Omega$ such, that $z \in K_{+}(p, \alpha, \beta) \cap \Omega, z \in \Omega$. Consequently there exists $w \in \partial \Omega$ such, that for every fixed $R>0, w \in \partial K_{+}(p, \alpha, \beta, R)$. Let us suppose, that point $w$ is first, except $p$, contained in $\partial K_{+}(p, \alpha, \beta, R)$, which means, that there were no other points from $\partial \Omega$ on $\partial K_{+}(p, \alpha, \beta, R)$.

Suppose that $w \notin \partial K_{+}(p, \alpha, \beta)$. Then $w \in \partial \mathbb{B}(p, R)$ and thus, there exists vicinity $U_{w} \subset K_{+}(p, \alpha, \beta)$. So, there is a point $v \in \Omega$ such that $v \in$ $\in U_{w}$. As $\Omega$ is starlike, $[0, v]$ is contained in $\Omega$. From the other hand

$$
[0, v] \cap K_{+}(p, \alpha, \beta, R) \neq \varnothing,
$$

which contradicts the fact that $K_{+}(p, \alpha, \beta, R) \subset \Omega^{\prime}$. 
So $w \in \partial K_{+}(p, \alpha, \beta)$. Through Theorem 1 there exists $\rho=\rho(p)$ such that cone $K_{-}(w, \alpha-\varepsilon, \beta-\varepsilon, \rho) \subset \Omega$ for every $\varepsilon \in(o, \min (\alpha, \beta))$.

In $\mathbb{C}$, we introduce polar coordinates 0 - pole, $\overrightarrow{0 p}$ - polar.

Consider the points $a_{\lambda}=p+(w-p) \lambda, \lambda \in(0,1)$. We show, that $a_{\lambda} \in K_{-}(w, \alpha-\varepsilon, \beta-\varepsilon, \rho)$ for sufficiently small $\rho_{\iota} 0$, when $\lambda$ close to 1 and $\varepsilon$ close to 0 . If this is true, then one the one hand $a_{\lambda} \in \Omega$, which follows from Theorem 1 , and on the other hand $a_{\lambda} \in \partial K_{+}(p, \alpha, \beta)$ since $w \in \partial K_{+}(p, \alpha, \beta)$. This contradiction get us that the theorem is true.

To prove the inclusion $a_{\lambda} \in K_{-}(p, \alpha-\varepsilon, \beta-\varepsilon, \rho)$ it is enough to show that

$$
-\frac{(\beta-\varepsilon) \pi}{2}<\arg \left(a_{\lambda}-w\right)-\arg (-w)<\frac{(\alpha-\varepsilon) \pi}{2} .
$$

Since $a_{\lambda}-w=p+(w-p) \lambda-w=(p-w)(1-\lambda),(3)$ can be rewritten as:

$$
-\frac{(\beta-\varepsilon) \pi}{2}<\arg (p-w)-\arg (-w)<\frac{(\alpha-\varepsilon) \pi}{2} .
$$

Here we get two ways:

1) Suppose that

$$
-\frac{\beta \pi}{2}<\arg (w)-\arg (p)<0
$$

this means that $\arg (w-p)-\arg (p)=-\frac{\beta \pi}{2}$.

We see that $\arg (p-w)-\arg (-w)=\arg (w-p)-\arg (w)$, so

$$
\arg (p-w)-\arg (-w)=-\frac{\beta \pi}{2}+\arg (p)-\arg (w)<0 .
$$

As $\arg (w)-\arg (p)<0$, for sufficiently small $\varepsilon>0$

$$
\arg (p)-\arg (w)>\frac{\varepsilon \pi}{2},
$$

and thus,

$$
\arg (p)-\frac{\beta \pi}{2}-\arg (w)>-\frac{\beta \pi}{2}+\frac{\varepsilon \pi}{2} .
$$

From inequality (5), it follows, that

$$
-\frac{(\beta-\varepsilon) \pi}{2}<\arg (p-w)-\arg (-w)<0 .
$$


2) Now let us suppose that

$$
0<\arg (w)-\arg (p)<\frac{\alpha \pi}{2} ;
$$

this means that $\arg (w-p)-\arg (p)=\frac{\alpha \pi}{2}$.

We see that $\arg (p-w)-\arg (-w)=\arg (w-p)-\arg (w)$ and so

$$
0<\arg (p-w)-\arg (-w)=\frac{\alpha \pi}{2}+\arg (p)-\arg (w) .
$$

As $\arg (w)-\arg (p)>0$, for sufficiently $\varepsilon>0$, one has

$$
\arg (w)-\arg (p)>\frac{\varepsilon \pi}{2},
$$

so that

$$
\frac{\alpha \pi}{2}+\arg (p)-\arg (w)<\frac{\alpha \pi}{2}-\frac{\varepsilon \pi}{2} .
$$

From (6), it follows, that

$$
0<\arg (p-w)-\arg (-w)<\frac{(\alpha-\varepsilon) \pi}{2} .
$$

Thus, from cases 1) and 2), it follows, that inequality (3) is true, and thus $a_{\lambda} \in K_{-}(w, \alpha-\varepsilon, \beta-\varepsilon, \rho)$ with $\lambda$ close enough to 1 . Hence we get a contradiction. The proof is completed.

Remark 1. Observe that $(\alpha, \beta)$-accessible domains are bounded, if $\alpha, \beta \in$ $\in(0,1)$, since these domains are $\alpha_{0}$-accessible, $\alpha_{0}=(\min (\alpha, \beta))$, and in [6] it was shown that $\alpha_{0}$-accessible domains are bounded for $\alpha_{0}>0$.

Theorem 3. If $\Omega \subset \mathbb{C}, 0 \in \Omega, \alpha, \beta \in(0,1)$, then the following assertions are equivalent:

(i) $\Omega$ is $(\alpha, \beta)$-accessible domain;

(ii) every unbounded cone $K_{+}(p, \alpha, \beta) \subset \Omega^{\prime}, p \in \partial \Omega$;

(iii) every unbounded cone $K_{+}(p, \alpha, \beta) \subset \Omega^{\prime}, p \in \Omega^{\prime}$;

(iv) for every point $p \in \partial \Omega$ and for every $\varepsilon \in(0, \min (\alpha, \beta))$ there exists an $r=r(p)>0$ such that the bounded cone $K_{-}(p, \alpha-\varepsilon, \beta-\varepsilon, r) \subset \Omega$.

Proof. In view of Theorems 1 and 2, it is sufficient to prove the implications $(i v) \Rightarrow(i)$ and $(i i) \Rightarrow(i i i)$.

Let $w=I(z)$ be the mapping inversion, defined as:

$$
w=\frac{1}{\bar{z}} .
$$


For the proof of $(i v) \Rightarrow(i)$, under this mapping, consider the image of the cone $K_{+}\left(p^{\prime}, \beta, \alpha\right) \backslash\left\{p^{\prime}\right\}$ to $K_{-}(p, \alpha, \beta)$, where $p=1 / \overline{p^{\prime}}$. Indeed, (7) is a bilinear mapping, having a circular feature and the property of preserving angles, so that the boundary of $K_{+}\left(p^{\prime}, \alpha, \beta\right)$ transfers into arcs, intersecting at points $p$ and 0 , and the angle of intersections of those circles at the point $p$ is $(\alpha+\beta) \pi / 2$, and the image will be lying inside intersection of these circles.

Now, let us consider the condition $(i v)$. Denote $G \subset \mathbb{C}$ as the image $I(\Omega \backslash 0)$. We will show that domain $G^{\prime}=\mathbb{C} \backslash G$ is $(\beta, \alpha)$-accessible.

To show this, we note that $0 \in G^{\prime}$, and for every point $p \in \partial \Omega$ there exists cone $K_{-}(p, \alpha-\varepsilon, \beta-\varepsilon, \rho) \subset \Omega$, for sufficient small $\rho=$ $=\rho(p, \varepsilon)>0$. Obviously, there exists a number $r=r(p, \rho)>0$ such that when $z=1 / \bar{w}$ follows the inclusion

$$
I\left(K_{+}\left(p^{\prime}, \beta-\varepsilon, \alpha-\varepsilon, r\right) \backslash\left\{p^{\prime}\right\}\right) \subset K_{-}(p, \alpha-\varepsilon, \beta-\varepsilon, \rho),
$$

which means that $I\left(K_{+}\left(p^{\prime}, \beta-\varepsilon, \alpha-\varepsilon, r\right) \backslash\left\{p^{\prime}\right\}\right) \subset \Omega$ for every point $p^{\prime} \in \partial G^{\prime}$. Thus $G^{\prime}$ is $(\beta-\varepsilon, \alpha-\varepsilon)$-accessible domain, $\varepsilon \in(0, \min (\alpha, \beta))$. From Theorem 2 it follows, that $K_{+}\left(p^{\prime}, \beta-\varepsilon, \alpha-\varepsilon\right) \subset G$. Passing to the limit $\varepsilon \rightarrow 0$ we get that $K_{+}\left(p^{\prime}, \beta, \alpha\right) \subset G^{\prime}$, so $G^{\prime}$ is $(\beta, \alpha)$-accessible. Hence, from Theorem 1, it follows that for every point $p \in \partial G^{\prime}$ and for every $\varepsilon \in(0, \min (\alpha, \beta))$ there exists an $r=r\left(p^{\prime}, \varepsilon\right)>0$ such that the cone $K_{-}\left(p^{\prime}, \alpha-\varepsilon, \beta-\varepsilon, r\right)$ belongs to $G^{\prime}$.

Note that under the mapping (7) the image of cone $K_{-}\left(p^{\prime}, \alpha-\varepsilon, \beta-\right.$ $-\varepsilon, r)$ belongs to $K_{+}(p, \alpha-\varepsilon, \beta-\varepsilon, R)$ for some $r=r(p, R)>0$, so that $I\left(K_{-}\left(p^{\prime}, \alpha-\varepsilon, \beta-\varepsilon, r\right)\right) \subset \Omega^{\prime}$. Hence and from definition we see that $\Omega$ is $(\alpha-\varepsilon, \beta-\varepsilon)$-accessible domain. Using Theorem 1 and allowing $\varepsilon \rightarrow 0$ we get, that $\Omega$ is $(\alpha, \beta)$-accessible. This proves the implication $(i v) \Rightarrow(i)$.

We now show, that if $\Omega$ satisfies the condition $(i i)$, then $\Omega$ satisfies the condition $($ iii $)$. Take arbitrary point $p \in \Omega^{\prime} \backslash \partial \Omega$. The segment $[0, p]$ intersects $\partial \Omega$. If this intersection has more than one point, then we take the closest to $p$ and denote it as $p^{\prime}$, and the next one - as $p^{\prime \prime}$. Then the cone $K_{+}\left(p^{\prime}, \alpha, \beta\right)$ contains inside sufficient small surroundings of point $p^{\prime \prime}$ and therefore points from $\Omega$. On the other hand, Theorem 2 says that $K_{+}\left(p^{\prime}, \alpha, \beta\right) \subset \Omega^{\prime}$. This is a contradiction the fact that $[0, p] \cap$ $\cap \partial \Omega=p^{\prime}$. Hence, from Theorem 2 , it follows that $K_{+}\left(p^{\prime}, \alpha, \beta\right) \subset \Omega^{\prime}$. We will now show, that $K_{+}(p, \alpha, \beta) \subset \Omega^{\prime}$. Indeed, since $|p|>\left|p^{\prime}\right|$, we have $K_{+}(p, \alpha, \beta)=K_{+}\left(p^{\prime}, \alpha, \beta\right)+\left(p-p^{\prime}\right)$, so that $\arg (p)=\arg \left(p^{\prime}\right)$. Then for every point $z \in K_{+}\left(p^{\prime}, \alpha, \beta\right)$, one has $z+\left(p-p^{\prime}\right) \in K_{+}(p, \alpha, \beta)$. Let us show 
that $z+\left(p-p^{\prime}\right)$ belongs to $K_{+}\left(p^{\prime}, \alpha, \beta\right)$. Since $z+\left(p-p^{\prime}\right) \in K_{+}(p, \alpha, \beta)$, we see that

$$
-\frac{\beta \pi}{2} \leq \arg \left(z+\left(p-p^{\prime}\right)-p\right)-\arg (p) \leq \frac{\alpha \pi}{2},
$$

and so, $\operatorname{as} \arg (p)=\arg \left(p^{\prime}\right)$,

$$
-\frac{\beta \pi}{2} \leq \arg \left(z-p^{\prime}\right)-\arg \left(p^{\prime}\right) \leq \frac{\alpha \pi}{2} .
$$

Hence, from definition of $K_{+}\left(p^{\prime}, \alpha, \beta\right)$, we obtain that $z+\left(p-p^{\prime}\right) \in$ $\in K_{+}\left(p^{\prime}, \alpha, \beta\right)$. Thus $K_{+}(p, \alpha, \beta) \subset \Omega^{\prime}$. Since the point $p \in \Omega^{\prime} \backslash \partial \Omega$ is arbitrary, we get the implication $(i i) \Rightarrow(i i i)$.

Remark 2. If $\left\{\Omega_{\gamma}\right\}$ is a family of $(\alpha, \beta)$-accessible domains, then the union $\Omega=\bigcup_{\gamma}\left\{\Omega_{\gamma}\right\}$ is also a $(\alpha, \beta)$-accessible domain. Actually, from Theorem 3, it follows that $\Omega$ is $(\alpha, \beta)$-accessible domain if and only if $K_{+}(p, \alpha, \beta) \cap \Omega=\varnothing$ for every point $p \in \Omega^{\prime}$. If $p \notin \Omega$, then $p \notin \Omega_{\gamma}$ for every $\gamma$. In this situation, $K_{+}(p, \alpha, \beta) \cap \Omega_{\gamma}=\varnothing$ for every $\gamma$. Thus, $K_{+}(p, \alpha, \beta) \cap\left(\bigcup \Omega_{\gamma}\right)=\varnothing$.

Theorem 4. If $\Omega$ is $(\alpha, \beta)$-accessible domain, $\alpha, \beta \in(0,1)$, then for every $\varepsilon \in(0, \min (\alpha, \beta))$ there exists an $R=R(\varepsilon)>0$ such that the cone $K_{-}(p, \alpha-\varepsilon, \beta-\varepsilon, R) \subset \Omega$ for every point $p \in \partial \Omega$.

Proof. From the implication $(i v) \Rightarrow(i)$ in proof of Theorem 3, it follows that for $(\alpha, \beta)$-accessible domains $\Omega$, the interior of complement $I\left(\Omega^{\prime}\right)=$ $=G^{\prime}$, using $z=I(w)=1 / \bar{w}$, is $(\beta, \alpha)$-accessible domain. Therefore it is enough to show that for every fixed $\varepsilon \in(0, \min (\alpha, \beta))$ there exists an $R=R(\varepsilon)>0$ such that for every point $p \in \partial \Omega$, the image of every $w \in$ $\in K_{-}(p, \alpha-\varepsilon, \beta-\varepsilon, R)$ using $z=I(w)$ considered inside $K_{+}\left(p^{\prime}, \beta, \alpha\right)$, $p^{\prime}=1 / \bar{p}$. Indeed, if it will be shown, then

$$
I\left(K_{-}(p, \alpha-\varepsilon, \beta-\varepsilon, R)\right) \subset G=I(\Omega) .
$$

Hence, as $I(w)$ is homeomorphism, we get $K_{-}(p, \alpha-\varepsilon, \beta-\varepsilon, R) \subset \Omega$.

Since $w \in K_{-}(p, \alpha-\varepsilon, \beta-\varepsilon, R), w=p+r e^{i(\phi+\arg (p))}, r \in(0, R]$,

$$
\phi \in((2-\beta+\varepsilon) \pi / 2,(2+\alpha-\varepsilon) \pi / 2),
$$

so that $\pi-\phi \in((\beta-\varepsilon) \pi / 2,(\alpha+\varepsilon) \pi / 2)$. 
By definition of the $K_{+}(p, \beta, \alpha)$, we get $I(w)=1 / \bar{w} \in \operatorname{Int} K_{+}\left(p^{\prime}, \beta, \alpha\right)$ if and only if

$$
-\frac{\alpha \pi}{2}<\arg \left(\frac{1}{\bar{w}}-\frac{1}{\bar{p}}\right)-\arg \left(\frac{1}{\bar{p}}\right)<\frac{\beta \pi}{2} .
$$

Now

$$
\begin{gathered}
\arg \left(\frac{1}{\bar{w}}-\frac{1}{\bar{p}}\right)-\arg \left(\frac{1}{\bar{p}}\right)=\arg \left(\frac{\overline{p-w}}{\overline{w p}}\right)-\arg (p)=\arg \left(\frac{\overline{p-w}}{\bar{w}}\right)= \\
=\arg \left(\frac{-r e^{-i(\phi+\arg (p))}}{\bar{p}+r e^{-i(\phi+\arg (p))}}\right)=\arg \left(e^{i(\pi-\phi-\arg (p))}\right)+\arg \left(p+r e^{i(\phi+\arg (p))}\right)= \\
=\pi-\phi-\arg (p)+\arg \left(p+r e^{i(\phi+\arg (p))}\right) .
\end{gathered}
$$

Since $0 \in \Omega$, we have $p \neq 0$. Then there exists an $R \in\left(0, \min _{p \in \partial \Omega}|p|\right)$ such that

$$
\left|\arg \left(p+R e^{i(\phi+\arg (p))}\right)-\arg (p)\right|<\frac{\varepsilon \pi}{2},
$$

therefore, for every $r \in(0, R)$ and for every $p \in \partial \Omega$, the following inequality holds:

$$
\left|\arg \left(p+r e^{i(\phi+\arg (p))}\right)-\arg (p)\right|<\frac{\varepsilon \pi}{2},
$$

thus the inequality (8) holds.

Hence we get that there exists an $R=R(\varepsilon)>0$ such that for every $p \in$ $\in \partial \Omega$, the image of the cone $K_{-}(p, \alpha-\varepsilon, \beta-\varepsilon, R)$ belongs to $\operatorname{Int}_{+}\left(p^{\prime}, \beta, \alpha\right)$. This proves the theorem.

Theorem 5. If a domain $\Omega \subset \mathbb{C}(\Omega \neq \mathbb{C})$ is $(\alpha, \beta)$-accessible, $\alpha, \beta \in$ $\in(0,1)$, then for every $\varepsilon \in(0, \min (\alpha, \beta))$ there exists an $R=R(\varepsilon)>0$ such that the cone $K_{-}(p, \alpha-\varepsilon, \beta-\varepsilon, R)$ belongs to $\Omega$ for every $p \in \bar{\Omega}$.

Proof. Assume that theorem is wrong. Then for some $\varepsilon \in(0, \min (\alpha, \beta))$ there exists sequence of points $w_{k} \in \Omega$ and a sequence of numbers $r_{k}$ such that the cone

$$
K_{-}\left(w_{k}, \alpha-\varepsilon, \beta-\varepsilon, r_{k}\right) \cap \Omega^{\prime} \neq \varnothing
$$

for every number $k \in \mathbb{N}$, and $r_{k} \rightarrow 0$. Since $\bar{\Omega}$ is compact, there exists a convergent subsequence of sequence $\left\{w_{k}\right\}$, that $w_{k}^{\prime} \rightarrow w_{0}^{\prime}$. Denote this subsequence as $\left\{w_{k}^{\prime}\right\}$. If $w_{0}^{\prime} \in \Omega$, then for sufficiently small $\rho>0$ ball 
$\mathbb{B}\left(w_{0}^{\prime}, \rho\right) \subset \Omega$. Starting from some number $k \geq N$, points $w_{k}^{\prime} \in \mathbb{B}\left(w_{0}^{\prime}, \rho\right)$, we have $K_{-}\left(w_{k}^{\prime}, \alpha-\varepsilon, \beta-\varepsilon\right) \cap \mathbb{B}\left(w_{0}^{\prime}, \rho\right) \subset \Omega$. Since the last fact contradicts (9), we get that $w_{0}^{\prime} \in \partial \Omega$.

Consider a sequence of points $p_{k} \in \partial \Omega, p_{k}=\lambda_{k} w_{k}^{\prime}, \lambda_{k}>1$. Note that $p_{k} \rightarrow w_{0}^{\prime}$ when $k \rightarrow \infty$ and $\lim _{k \rightarrow \infty} p_{k}=p_{0}=w_{0}^{\prime}$. Indeed, if it is wrong, then $p_{0}=\lambda_{0} w_{0}^{\prime}, \lambda_{0}>0$ and $\lambda \neq 1$. Since $p_{0} \in \partial \Omega$, for every surroundings $U_{p_{0}}: U_{p_{0}} \cap \Omega \neq \varnothing$. On the one hand $\Omega$ is $(\alpha, \beta)$-accessible domain and $w_{0}^{\prime} \in \partial \Omega$, so the cone $K_{+}\left(w_{0}^{\prime}, \alpha, \beta\right)$ belongs to $\Omega^{\prime}$. On the other hand, since $\left|p_{0}\right|>\left|w_{0}^{\prime}\right|$, the sufficient small surroundigs $U_{p_{0}} \subset K_{+}\left(w_{0}^{\prime}, \alpha, \beta\right)$, so that $K_{+}\left(w_{0}^{\prime}, \alpha, \beta\right) \cap \Omega \neq \varnothing$, but this can not be true (see Theorem 2). Hence we get that $p_{0}=w_{0}^{\prime}$.

Since $\lim _{k \rightarrow \infty} w_{k}^{\prime}=p_{0}=\lim _{k \rightarrow \infty} p_{k}, p_{k}=\lambda_{k} w_{k}^{\prime}, \lambda_{k} \rightarrow 1^{+}$as $k \rightarrow \infty$. Therefore for number $R$ from Theorem 4 and for sufficient large number $k$, points $w_{k}^{\prime} \in K_{-}\left(p_{k}, \alpha-\varepsilon, \beta-\varepsilon, R\right)$ and

$$
K_{-}\left(w_{k}^{\prime}, \alpha-\varepsilon, \beta-\varepsilon, r_{k}^{\prime}\right) \subset K_{-}\left(p_{k}, \alpha-\varepsilon, \beta-\varepsilon, R\right) .
$$

By Theorem 4 , the cone $K_{-}\left(p_{k}, \alpha-\varepsilon, \beta-\varepsilon, R\right) \subset \Omega$ for some fixed $R=$ $=R(\varepsilon)>0$, so that $K_{-}\left(w_{k}^{\prime}, \alpha-\varepsilon, \beta-\varepsilon, r_{k}^{\prime}\right) \subset \Omega$. The last contradicts the relation (9). Theorem 5 is proved.

3. Case of domains with smooth boundary. Here we assume that the domain $\Omega \subset \mathbb{R}^{2}$ has smooth boundary $\partial \Omega$ given by equation:

$$
F(x, y)=0
$$

and

$$
F(x, y)<0
$$

is $\Omega$.

Smooth function $F(x, y)$ can be set locally which means that $F(x, y)=$ $=F_{p}(x, y)$ in the neighborhood of each point $p \in \partial \Omega$. Since $\partial \Omega$ in the neighborhood of each point $p \in \partial \Omega$ can be defined by the equation:

$$
x=f(y) \text { or } y=f(x),
$$

we can assume that $\operatorname{grad} F(p) \neq 0$ for every point $p \in \partial \Omega$.

Denote by $n(p)=\frac{\operatorname{grad} F(p)}{\|\operatorname{grad} F(p)\|}$, the external unit normal vector at point $p \in \partial \Omega$. 
The following lemma is a consequence of the lemma from [6].

Lemma 1. Let $\Omega \subset \mathbb{C}$ with smooth boundary $\partial \Omega$, and $n(p)$ is external normal vector at point $p \in \partial \Omega$. Then for every fixed $\alpha, \beta \in(0,1)$ there exists $r>0$ such that

$$
\begin{gathered}
K^{+}(p, \alpha, \beta, r)= \\
=\left\{z \in \mathbb{C}:-\frac{\beta \pi}{2}<\arg (z-p)-\arg (n(p))<\frac{\alpha \pi}{2},\|z-p\|<r\right\} \subset \Omega^{\prime}, \\
K^{-}(p, \alpha, \beta, r)= \\
=\left\{z \in \mathbb{C}:-\frac{\beta \pi}{2}<\arg (z-p)-\arg (-n(p))<\frac{\alpha \pi}{2},\|z-p\|<r\right\} \subset \Omega .
\end{gathered}
$$

Theorem 6. Let $\Omega \in \mathbb{C}, \partial \Omega$ be smooth boundary. Then for every fixed $\alpha, \beta \in(0,1)$ domain $\Omega$ is $(\alpha, \beta)$-accessible if and only if

$$
-\frac{(1-\beta) \pi}{2} \leq \arg (p)-\arg (n(p)) \leq \frac{(1-\alpha)}{2}
$$

for every point $p \in \partial \Omega$.

Proof. Suppose that $\Omega$ is $(\alpha, \beta)$-accessible. We will show that the inequality (10) holds. As $\Omega$ is $(\alpha, \beta)$-accessible, it is starlike with respect to 0 , and under our assumptions about $F(z)$ it follows from [7] that $\Omega$ starlike if and only if $\left(\frac{p}{\|p\|}, \frac{\operatorname{grad}(F(p))}{\|\operatorname{grad} F(p)\|}\right) \geq 0$ for every $p \in \partial \Omega$. Indeed, $\frac{\operatorname{grad} F(p)}{\|\operatorname{grad} F(p)\|}=n(p)$ is external normal vector at point $p$ and

$$
\left(\frac{p}{\|p\|}, \frac{n(p)}{\|n(p)\|}\right) \geq 0 \Leftrightarrow \cos \phi_{p} \geq 0
$$

which means that $\left|\phi_{p}\right| \leq \pi / 2$. Let $\phi_{p}=\arg (p)-\arg (n(p)), \arg (p) \in$ $\in[0,2 \pi] . \arg (p)$ increases when crawling $\partial \Omega$ in positive direction, and $\arg (n(p))$ changes continuously with a continuous changing of $p \in \partial \Omega$. Suppose that at point $p$ the inequality (10) doesn't hold, then we get:

$$
\frac{(1-\alpha) \pi}{2}<\arg (p)-\arg (n(p)) \leq \frac{\pi}{2},
$$

or

$$
-\frac{\pi}{2} \leq \arg (p)-\arg (n(p))<-\frac{(1-\beta) \pi}{2} .
$$


For simplicity, we assume that $\arg (p)=0, p \in \mathbb{R}$ (this could be achieved by converting the rotation on which the domain $\Omega$ is not sensitive). Thus

$$
-\frac{\pi}{2} \leq \arg (n(p))<\frac{(1-\alpha) \pi}{2}
$$

or

$$
\frac{(1-\beta) \pi}{2}<\arg (n(p)) \leq \frac{\pi}{2} .
$$

As $\Omega$ is $(\alpha, \beta)$-accessible, the cone $K_{+}(p, \alpha, \beta) \subset \Omega^{\prime}$. Let

$$
\begin{gathered}
K^{-}(p, \gamma, m)= \\
=\left\{z \in \mathbb{C}:-\frac{\gamma \pi}{2} \leq \arg (z-p)-\arg (-n(p)) \leq \frac{\gamma \pi}{2},|z-p|<m\right\} .
\end{gathered}
$$

From a lemma proved in [6], it follows that for every fixed $\gamma \in(0,1)$ there exists an $m>0$ such that $K^{-}(p, \gamma, m) \subset \Omega$. Take a point $z \in$ $\in \partial K_{+}(p, \alpha, \beta, r), z=p+\rho e^{i \phi}, \phi=\{\alpha \pi / 2,-\beta \pi / 2\}, 0<\rho<r$.

Separately consider the cases $\left(13^{*}\right),\left(14^{*}\right)$.

1) Case $\left(13^{*}\right)$. Let $z^{+}=p+\rho e^{i \alpha \pi / 2}$. We will show that $z^{+}$belongs to $K^{-}(p, \gamma, m)$ if $\rho<m$. Choose $\arg (-n(p))$ such that

$$
\arg (-n(p))=\pi+\arg (n(p)) .
$$

Then

$$
\frac{\pi}{2} \leq \arg (-n(p))<\frac{(1+\alpha) \pi}{2} .
$$

Since $\arg \left(z^{+}-p\right)=\alpha \pi / 2$, one has

$$
-\frac{\pi}{2}<\arg \left(z^{+}-p\right)-\arg (-n(p)) \leq-\frac{(1-\alpha) \pi}{2} .
$$

From (16) it follows that for sufficiently small $m>0$ there exists $\gamma \in(0,1)$ with

$$
-\frac{\gamma \pi}{2}<\arg \left(z^{+}-p\right)-\arg (-n(p)) \leq \frac{\gamma \pi}{2} .
$$

Last inequality means that $z^{+} \in K^{-}(p, \gamma, m)$ with $\rho<m$. A lemma from [6] guarantees that $K^{-}(p, \gamma, m) \subset \Omega$ and thus $z^{+}$also belongs $\Omega$, which contradicts the fact that $z^{+} \in \partial K_{+}(p, \alpha, \beta) \subset \Omega^{\prime}$.

2) Case $\left(14^{*}\right)$. Now let $z^{-}=p+\rho e^{-i \frac{\beta \pi}{2}}$. We will show that $z^{-}$belongs to $K^{-}(p, \gamma, m)$ if $\rho<m$. Choose $\arg (-n(p))$ such that

$$
\arg (-n(p))=\arg (n(p))-\pi .
$$


Then

$$
-\frac{(1+\beta) \pi}{2}<\arg (-n(p)) \leq-\frac{\pi}{2} .
$$

Since $\arg \left(z^{-}-p\right)=-\beta \pi / 2$, we have

$$
\frac{(1-\beta) \pi}{2} \leq \arg \left(z^{-}-p\right)-\arg (-n(p))<\frac{\pi}{2} .
$$

From (18) it follows that for sufficiently small $m>0$ there exists $\gamma \in(0,1)$ with

$$
-\frac{\gamma \pi}{2}<\arg \left(z^{-}-p\right)-\arg (-n(p)) \leq \frac{\gamma \pi}{2} .
$$

Last inequality means, that $z^{-} \in K^{-}(p, \gamma, m)$ with $\rho<m$. A lemma from [6] guarantees that $K^{-}(p, \gamma, m) \subset \Omega$. Thus $z^{-}$also belongs to $\Omega$, which contradicts the fact that $z^{-} \in \partial K_{+}(p, \alpha, \beta) \subset \Omega^{\prime}$.

Contradictions in cases 1$)$ and 2$)$ mean that if $\Omega$ is $(\alpha, \beta)$-accessible domain then inequality (10) holds.

Now let for every point $p \in \partial \Omega$ the inequality (10) hold. We show that $\Omega$ is $(\alpha, \beta)$-accessible domain. At first show that $\Omega$ is $(\eta, \theta)$-accessible domain for $\eta \in(0, \alpha), \theta \in(0, \beta)$, i. e. for every point $p \in \partial \Omega$ the cone $K_{+}(p, \eta, \theta, r) \subset \Omega^{\prime}, r=r(p)>0$. Fix $p$ and take a point $z \in K_{+}(p, \eta, \theta, r)$ with sufficiently small $r$ then

$$
-\frac{\theta \pi}{2} \leq \arg (z-p)-\arg (p) \leq \frac{\eta \pi}{2} .
$$

Compose (10) and (19):

$$
-\frac{(1-\beta+\theta) \pi}{2} \leq \arg (z-p)-\arg (n(p)) \leq \frac{(1-\alpha+\eta) \pi}{2} .
$$

Last inequality means that $z$ belongs to $K^{+}(p, 1-\alpha+\eta, 1-\beta+\theta, r)$. Denote $\psi=\max (1-\alpha+\eta, 1-\beta+\theta), \psi \in(0,1)$. Then

$$
K^{+}(p, 1-\alpha+\eta, 1-\beta+\theta, r) \subset K^{+}(p, \psi, r) .
$$

A lemma from [6] guarantees that $K^{+}(p, \psi, r) \subset \Omega^{\prime}$ for sufficiently small $r>0$, and thus $K^{+}(p, 1-\alpha+\eta, 1-\beta+\theta, r) \subset \Omega^{\prime}$.

We get that $z \in \Omega^{\prime}$ for every point $z \in K_{+}(p, \eta, \theta, r)$ with sufficiently small $r>0$. Thus $\Omega$ is $(\eta, \theta)$-accessible domain. Now, applying Theorem 
2 and passing to a limit $\eta \rightarrow \alpha, \theta \rightarrow \beta$ we get that $\Omega$ is a $(\alpha, \beta)$-accessible domain. Theorem 6 proved.

Corollary 1. Denote by $e$ the symmetry axis of the cone $K_{+}(p, \alpha, \beta)$. Then a condition (10) is equivalent to

$$
\left(\frac{e}{\|e\|}, n(p)\right) \geq \sin \left(\frac{(\alpha+\beta) \pi}{4}\right) .
$$

Proof. Fix $p \in \partial \Omega$. With rotation transformation, assume that $\arg (p)=0$. The solution of the cone $K_{+}(p, \alpha, \beta, r)$ is $\frac{(\alpha+\beta) \pi}{2}$. Note that $\arg (e) \in$ $\in\left(-\frac{\pi}{4}, \frac{\pi}{4}\right)$. Then

$$
\frac{\alpha \pi}{2}-\arg (e)=\frac{(\alpha+\beta) \pi}{4} i f \arg (e) \geq 0,
$$

or

$$
\frac{\beta \pi}{2}+\arg (e)=\frac{(\alpha+\beta) \pi}{4} \text { if } \arg (e)<0 .
$$

From the last inequalities we get $\arg (e)=\frac{(\alpha-\beta) \pi}{4}$. Thus from (10) we get:

$$
-\frac{(2-\alpha-\beta) \pi}{4} \leq \arg (e)-\arg (n(p)) \leq \frac{(2-\alpha-\beta) \pi}{4},
$$

and this is equivalent:

$$
\left(\frac{e}{\|e\|}, n(p)\right) \geq \sin \left(\frac{(\alpha+\beta) \pi}{4}\right) .
$$

The following theorem gives a sufficient condition for $(\alpha, \beta)$-accessible domains. Here $A^{*}$ denotes a matrix, conjugate to a matrix $A$. Let $e$, as in corollary to Theorem 6 , be a vector lying on the symmetry axis of the cone $K_{+}(p, \alpha, \beta)$.

Theorem 7. Let $\Omega \subset \mathbb{R}^{2}$ be a bounded domain with $0 \in \Omega, \alpha, \beta \in(0,1)$. Let $f=\left(\begin{array}{l}u \\ v\end{array}\right)$ be a diffeomorphism of a domain $\Omega$ at the unit circle centered at the point $0, f(0)=0$, and $D f(x)$ is nonsingular differential in 
every point $x \in \Omega$.

If for a number $\delta>0$ the inequality:

$$
\frac{f^{*}(x) D f(x) e}{\left\|f^{*}(x) D f(x)\right\|\|e\|} \geq \sin \left(\frac{(\alpha+\beta) \pi}{4}\right)
$$

holds in $\Omega(\delta)=\{x \in \Omega: \operatorname{dist}(x, \partial \Omega)<\delta\}$, then $\Omega$ is $(\alpha, \beta)$-accessible domain.

Proof. Denote by $\Omega_{r}=\left\{x \in \Omega: u^{2}+v^{2}<r^{2}\right\}$ with $r \in(0,1) . \Omega_{r} \subset \Omega$ and $\partial \Omega_{r}-$ smooth boundary, given by equation:

$$
F(x)=u^{2}+v^{2}-r^{2}=0 .
$$

Since $D f(x)$ is nonsingular for every $x \in \Omega$, then $f^{*}(p) D f(p) \neq 0$ for every point $p \in \partial \Omega_{r}$. Note that

$$
\begin{gathered}
f^{*}(x) D f(x)=(u, v)\left(\begin{array}{cc}
\frac{\partial u}{\partial x_{1}} & \frac{\partial u}{\partial x_{2}} \\
\frac{\partial v}{\partial x_{1}} & \frac{\partial v}{\partial x_{2}}
\end{array}\right)= \\
=\left(u \frac{\partial u}{\partial x_{1}}+v \frac{\partial v}{\partial x_{1}}, u \frac{\partial u}{\partial x_{2}}+v \frac{\partial v}{\partial x_{2}}\right)=\left(\frac{1}{2} \frac{\partial}{\partial x_{1}}\left(u^{2}+v^{2}\right), \frac{1}{2} \frac{\partial}{\partial x_{2}}\left(u^{2}+v^{2}\right)\right)= \\
=\frac{1}{2} \operatorname{gradF}(x) .
\end{gathered}
$$

Then $\operatorname{grad} F(p)=2 f^{*}(p) D f(p) \neq 0$ for every point $p \in \partial \Omega$.

As $\Omega$ is bounded, for fixed $\delta>0 \partial \Omega_{r} \subset \Omega(\delta)$ for $r \in\left(r_{0}, 1\right)$, with $r_{0}$ sufficiently close to 1 .

By the condition in Theorem 7 we get,

$$
\begin{aligned}
\left(\frac{e}{\|e\|}, \frac{\operatorname{grad} F^{*}(x)}{\left\|\operatorname{grad} F^{*}(x)\right\|}\right) & =\frac{\operatorname{grad} F(x) e}{\|\operatorname{grad} F(x)\|\|e\|}=\frac{f^{*}(x) D f(x) e}{\left\|f^{*}(x) D f(x)\right\|\|e\|} \geq \\
& \geq \sin \left(\frac{(\alpha+\beta) \pi}{4}\right) .
\end{aligned}
$$

Now, from the corollary after Theorem 6 we get that $\Omega_{r}$ is $(\alpha, \beta)$-accessible domain, and from remark after Theorem 3 it follows that $\Omega=\bigcup_{r \in\left(r_{0}, 1\right)} \Omega_{r}$ is $(\alpha, \beta)$-accessible. Thus, Theorem 7 is proved.

Acknowledgment. This work was supported by the Russian Foundation for Basic Research (project No. 14-01-00510) and by Program of Strategic Development of Petrozavodsk State University. 


\section{References}

[1] Besov O. V., Il'in V. P., Nikolskii S. M. Integral presentation of functions and theorem of embanding. M., Nauka, 1975.

[2] Dolzenko E. P. Boundary properties of arbitrary functions. (In Russian) Proc. of USSR. Ac. of Sci. Ser. Math., 1967, vol. 31, no. 1, pp. 3-14. DOI: 10.1070/IM1967v001n01ABEH000543.

[3] Math. encyclopedia. Moscow, 1979, vol. 2.

[4] Zaremba S. Sur le principe de Dirichlet. Acta Math., 1911, vol. 34, pp. 293-316. DOI: 10.1007/BF02393130.

[5] Anikiev A.N. Plane domains with special cone condition. Russian Mathematics, 2014, vol. 58, no. 2, pp. 62-63. DOI: 10.3103/S1066369X14020108.

[6] Liczberski P., Starkov V. V. Domains in $\mathbb{R}^{n}$ with conical accessible boundary. J. Math. Anal. Appl., 2013, vol. 408, no. 2, pp. 547-560.

[7] Liczberski P., Starkov V. V. Planar $\alpha$-angularly starlike domains, $\alpha$-angularly starlike functions and their generalizations to multidimensional case. International Conference "60 Years of analytic functions in Lublin", 2012, pp. 117-125.

Received July 07, 2014.

In revised form, October 13, 2014.

Petrozavodsk State University

33, Lenina st., 185910 Petrozavodsk, Russia

E-mail: anikiev_a@mail.ru 